\title{
Infertility as a Cancer Risk Factor - A Review
}

\author{
I. Cetin*, V. Cozzi, P. Antonazzo \\ Obstetrics and Gynecology Department of Mother and Child Hospital Luigi Sacco, University of Milano, via GB Grassi 74, 20157 Milano, Italy
}

\section{A R T I C L E I N F O}

Article history:

Accepted 8 August 2008

Available online xxx

\section{Keywords:}

Infertility

Cancer risk

Fertility drugs

\begin{abstract}
A B S T R A C T
Ovarian, endometrial and breast cancers are associated with several risk factors, such as low parity, infertility, early age at menarche, and late age at menopause. Frequently most of these risk factors coexist in infertile patients and some studies suggested that the different infertility causes can be involved in cancer risk development. In particular case-control and cohort studies investigated the possible role of ovulatory disorders, endometriosis and unexplained infertility in increasing the risk of this disease. Most studies have shown no overall increased risk in invasive ovarian cancer in subfertile patients, although nulliparity has been consistently associated with increased rates of ovarian tumor, in particular with borderline and endometrioid cancers in patients with a history of endometriosis. Different studies reported that infertile women are not at risk for breast cancer. However, women affected by infertility may be more at risk for endometrial cancer, particularly if affected by ovulatory disorders.

Moreover, infertility is now often treated with medical devices that could by themselves modify the hormonal environment and be cofactors in the cellular changes towards cancer development. However, although early studies suggested that infertility medications were associated to increased risk in ovarian cancer, subsequent studies have been mainly reassuring, although suggesting that type and duration of medical treatment can increase the malignancy risk.

An increased risk of endometrial cancer in patients undergoing infertility treatment has been reported, as expected by the similar structure shared by clomiphene and tamoxiphene.

Since breast cancer is widely recognized as having a hormonal etiology, a possible role of fertility medications to promote cancer has been hypothesized. However, many large studies were not able to find an associated risk of breast cancer.

In conclusion, nowadays, firm answers about the precise effects of infertility and its treatment on cancer risk are not available but findings are generally reassuring. Further studies about fertility drug treatments on larger populations may offer in the future longer follow-up and more precise data with better adjustments for confounding factors.
\end{abstract}

(c) 2008 Published by Elsevier Ltd.

Infertility is a condition defined by the inability to conceive, or to get pregnant, within 1 year. Infertility has been recognized by the WHO as a problem affecting between $15 \%$ and $20 \%$ of couples in developed countries. Causes affecting the female are involved in $35-40 \%$ of cases. However, infertility is not a disease in itself, rather, it can be the result of many different disorders, from malformative, to endocrine, autoimmune, infective as well as psychological. Since these factors involve the female reproductive system, concerns have developed about the future health of these women, specifically whether infertility would represent a risk factor for future cancer development. Moreover, infertility is now often treated with medications and procedures that could by themselves modify the hormonal environment and be cofactors in the cellular changes towards cancer development.

\footnotetext{
* Corresponding author. Tel.: +39 02 50319804; fax: +39 0250319805

E-mail address: irene.cetin@unimi.it (I. Cetin).
}

This review addresses the potential association between infertility and cancer development in females evaluated by both cohort and case-control studies. Moreover, it focuses on the influence of infertility treatment such as ovulation induction.

\section{Ovarian cancer and infertility}

Nulliparity itself represents a risk factor for epithelial ovarian cancer. If infertility per se could be a condition associated with $\mathbf{Q 4}$ increased risk of ovarian cancer has been investigated by a significant number of cohort and case-control studies that are summarized in Tables 1 and 2.

Numerous cohort studies have compared ovarian cancer rates in subfertile women with those of the general population, using the standardized incidence ratio (SIR) [1-7]. The potential limitation of most of these studies is the small number of ovarian cancers and the potential confounding factor of nulliparity. A higher incidence in infertile women could indeed be attributable to nulliparity itself, 
Table 1

Selected cohort studies studying the association between infertility (part A), fertility drugs (part B) and ovarian cancer risk

\begin{tabular}{|c|c|c|c|c|}
\hline \multicolumn{5}{|c|}{ Standardized incidence ratios ( $95 \%$ CIs) vs general population } \\
\hline \multicolumn{5}{|c|}{ Infertility and ovarian cancer risk } \\
\hline Brinton et al. [1] & 2335 & 11 & Evaluated for infertility & 1.28 (not given) \\
\hline Modan et al. [4] & 2496 & 12 & Infertility patients treated & $1.6(0.8-2.9)$ \\
\hline Venn et al. [2] & 29,700 & 13 & IVF patients evaluated & $0.99(0.57-1.70)$ \\
\hline Potashnik et al. [3] & 1197 & 2 & Evaluated for infertility & $0.91(0.1-3.27)$ \\
\hline Rossing et al. [6] & 3837 & 11 & Evaluated for infertility & $2.5(1.3-4.5)$ \\
\hline Brinton et al. [45] & 12,193 & 45 & Evaluated for infertility & $1.98(1.4-2.6)$ \\
\hline \multicolumn{5}{|c|}{ Fertility drugs and ovarian cancer risk } \\
\hline \multirow[t]{3}{*}{ Rossing et al. [6] } & 3837 & 11 & No drug & $1.4(0.2-5.0)$ \\
\hline & & & Clomiphene & $3.1(1.4-5.9)$ \\
\hline & & & hMG/FSH & $5.6(0.1-31.0)$ \\
\hline \multirow[t]{3}{*}{ Modan et al. [4] } & 2496 & 12 & No drug & $1.6(0.6-3.5)$ \\
\hline & & & All treatments & $1.7(0.6-3.8)$ \\
\hline & & & Clomiphene & $2.7(0.9-5.8)$ \\
\hline \multirow[t]{2}{*}{ Doyle et al. [52] } & 5556 & 6 & No treatment & $1.7(0.2-6.0)$ \\
\hline & & & Treatment & $0.6(0.2-2.2)$ \\
\hline \multirow[t]{2}{*}{ Brinton et al. [45] } & 12,193 & 45 & No clomiphene & $2.1(1.4-3.0)$ \\
\hline & & & Clomiphene & $1.8(1.0-3.0)$ \\
\hline \multirow[t]{2}{*}{ Klip et al. [46] } & 23,592 & 15 & No IVF & $1.4(0.4-3.2)$ \\
\hline & & & IVF & $1.4(0.7-2.6)$ \\
\hline
\end{tabular}

rather than to the infertile condition. The incidence of ovarian cancer has been reported to be similar to the age-adjusted general population incidence in all except for three large studies [5-8]. Brinton et al. in a cohort of US women [5], Venn et al. in a cohort of Australian women [2], Potashnik et al. [3] and Modan et al. [4] in cohorts of Israeli Women found no increased risk of ovarian cancer compared to the general population with an average of follow-up at least of 10 years [1-4]. An increased risk of ovarian cancer was instead reported by Brinton et al. (SIR 1.98, 95\% CI 1.4-2.6) Rossing et al. (SIR 2.5, 95\% CI 1.3-4.5) and recently by Tworoger et al. (Rate ratio $=1.36$, 95\% CI: 1.07-1.75) [5-8]. The inclusion of borderline tumors, which are known to be less aggressive and to have a better prognosis than other malignant tumors, as well as invasive tumors may explain the different data reported in Rossing's study. Other studies have reported borderline tumors to be increased in infertile women [7]. Their increased detection in infertile women seeking for infertility investigations may reflect selection biases rather than a true increased incidence. Parity status adjustment which is the most relevant confounding factor has been recently reported by Jensen et al. [9]. This recent and large cohort study, including 54,362 women with diagnosis of infertility who were referred to Danish fertility clinics between 1963 and 1998, using parity specific cancer incidence, revealed a significantly increased SIR for ovarian cancer $(1.46,95 \% \mathrm{CI}: 1.24-1.71)$ [9].

Some cohort studies offer internal comparisons within the cohort of infertile women, which do allow adjustment for important ovarian cancer risk predictors and try to clarify the real effects of different and independent causes of infertility. After correcting for age at menarche, breastfeeding, use of ovulation therapy, tubal ligation, hysterectomy, age at menopause, menopausal hormone use, Brinton et al. report no increased ovarian cancer risk associated with ovulatory causes of infertility, fallopian tube dysfunction, or male factor/mechanical subfertility, while endometriosis associated to primary infertility showed an RR of 2.27 [5]. Ovarian tumors were roughly twice as likely to develop in women with ovulatory abnormalities than in the infertile women with other types of abnormalities [6].

Case-control studies have generally reported subfertility to be weakly, but not significantly, associated with increased rates of ovarian cancer in nulligravid or nulliparous women, but not in women who have ever been pregnant [10-12]. The most recent well-conducted case-control study by Rossing et al. reported a nonsignificantly increased risk of epithelial ovarian cancer in nulliparous (OR $1.3,95 \% \mathrm{CI} 0.7-2.5$, NS) but not in parous women with a history of subfertility [12]. Whittemore et al. [10] and Ness et al. [11] conducted the largest pooled analysis of several case-control studies. Whittemore et al. considered in the analysis 12 US casecontrol studies carried out between 1957 and 1985, totalizing 2197 cases of ovarian cancer and 4144 controls with a history of subfertility [10]. The analysis revealed a higher but not significantly increased risk of ovarian cancer in nulligravid subfertile women than in gravid subfertile women (OR 1.4, 95\% CI 0.86-2.3, NS vs OR $0.87,95 \%$ CI $0.67-1.1, \mathrm{NS}$ ). Ness et al. considered in the analysis eight case-control studies, summing up to 5207 cases of ovarian cancer

Table 2

Case-control studies studying the association between infertility (part A), fertility drugs (part B) and ovarian cancer risk

\begin{tabular}{|c|c|c|c|c|}
\hline Authors & No. of cases & No. of controls & Comparison & OR $(95 \% \mathrm{CI})$ \\
\hline \multicolumn{5}{|c|}{ Infertility and ovarian cancer risk } \\
\hline Whittemore et al. [10] & 2197 & 8893 & 12 US case-control studies (1956-1986) & $\begin{array}{l}\text { Nulligravid: } 1.4(0.86-0.23) \\
\text { Gravid: } 0.87(0.67-1.1)\end{array}$ \\
\hline Ness et al. [11] & 5207 & 7705 & Eight case-control studies (1989-1999) & $\begin{array}{l}\text { Nulligravid: } 1.1(0.91-1.55) \\
\text { Gravid: } 1.1(1.02-1.31)\end{array}$ \\
\hline \multicolumn{5}{|c|}{ Fertility drugs and ovarian cancer risk } \\
\hline Shu et al. [47] & 229 & 229 & Drugs vs no drugs use & $2.1(0.2-22.7)$ \\
\hline Whittemore et al. [10] & 718 & 1236 & Fertility drugs use vs no infertility & $2.8(1.3-6.1)$ \\
\hline Franceschi et al. [48] & 195 & 1339 & Drugs vs no drugs use & $0.7(0.2-3.3)$ \\
\hline Ness et al. [11] & 149 & 911 & Drugs vs no drugs use & $0.93(0.7-1.2)$ \\
\hline Parazzini et al. [49] & 971 & 2758 & $\begin{array}{l}\text { Drugs vs no drugs use } \\
\geq 6 \text { cycles }\end{array}$ & $\begin{array}{l}1.1(0.4-3.3) \\
1.0(0.2-3.8)\end{array}$ \\
\hline
\end{tabular}


and 7705 controls [11]. Overall, women seeking medical attention for infertility had no increased risk of ovarian cancer. The subanalysis about nulligravid and gravid women found a weak association between potentially subfertile women with epithelial ovarian cancer in gravid (OR 1.16, 95\% CI 1.02-1.31) but not in nulligravid women (OR $1.19,95 \% \mathrm{CI} 0.91-1.55 \mathrm{NS}$ ). It is interesting to note that both meta-analysis revealed an increased rate of ovarian cancer in women with prolonged infertility: Whittemore et al. reported that nulligravid and gravid women with a history of more than 15 years of unprotected intercourse had increased risk of developing ovarian cancer compared to those with less than 2 years of unprotected intercourse. Similarly, Ness et al. found that nulligravid women with more than 5 years of baby seeking presented a threefold increased risk of ovarian cancer than women who had attempted for less than 1 year (OR 2.67, 95\% CI 1.91-3.74) [10,11].

Some studies have also evaluated the specific cause of infertility and the correlated risk of ovarian cancer $[10,11,13]$. Ovulatory disorders, endometriosis, and unexplained infertility are the most common diagnoses associated to ovarian cancer. Schildkraut et al. reported a 2.4-fold (95\% CI 1.0-5.9) increased risk in women affected by polycystic ovary syndrome (PCOS) [13], while Whittemore et al. and Ness et al. in the sub-analysis of risk in women with infertility linked to ovulatory disorders did not report an increased risk of ovarian cancer $[10,11]$. Unexplained infertility was reported to be an independent risk factor for ovarian cancer in different reports $[2,14]$ and in the meta-analysis by Ness et al. [11], but not by Whittemore et al. [10].

Endometriosis is the subtype of infertility with more agreement between cohort and case-control studies associated to cancer risk. Since endometriosis is a condition associated to infertility, this relationship should be pointed out with caution considering that the increased cancer risk may be due to nulliparity and not to endometriosis itself. However, studies that found a relationship were able to adjust for the effects of parity, showing these relationships to be independent of each other [15]. Brinton et al. reported that women affected by endometriosis presented a twofold risk of developing ovarian cancer than the general population in a large cohort of 20,686 women hospitalized for endometriosis from 1969 to 1983 based on the National Swedish Cancer Registry (SIR 1.9, 95\% CI 1.3-2.8) [16]. The risk of ovarian cancer was particularly increased among women with a long-standing history of ovarian endometriosis ( $>10$ years) (SIR 4.2 95\% CI 2.0-7.7). The prolongation of this study, which enrolled 64,492 women from 1969 to 2000 still confirmed this result (SIR 1.4; 95\% CI 1.2-1.7) [17].

A recent report by Brinton et al. [1] evaluated the risk related to endometriosis according to the different types of subfertility in a large cohort of 12,193 women recruited in the US from 1965 to 1988. Overall infertile patients presented a significantly higher risk of ovarian cancer ( $\mathrm{SIR}=2.0 ; 95 \% \mathrm{CI} 1.4-2.6)$ with the risk for patients with primary infertility ( $\mathrm{SIR}=2.7 ; 95 \% \mathrm{CI} 1.8-4.0$ ) higher than for patients with secondary infertility ( $\mathrm{SIR}=1.4 ; 95 \% \mathrm{CI} 0.9$ 2.3). Amongst infertile women, patients affected by endometriosis had the highest risk with an SIR of 2.5 (95\% CI 1.3-4.2) compared to the general population and an SIR of 4.2 (95\% CI 2.0-7.7) for the group with primary infertility. Comparing the causes of infertility, the SIR in women with endometriosis was 1.3 (95\% CI 0.6-2.6). When restricting the analysis to endometriosis and primary infertility the SIR reached the value of 2.7 (1.1-6.7). At variance, a recent Swedish cohort study by Melin et al., conducted on 63,630 women and 3822 cases of cancer, investigated the risk of cancer in endometriosis stratifying for parity and reported different data [15]. An increased risk of ovarian cancer with an SIR of 1.36 was reported, but no significant differences between parous and nulliparous women with endometriosis, and a nonsignificant decrease in the risk of ovarian cancer with increasing parity. This study shows that the lack of parity is not the cause of the increased ovarian cancer in this population: other risk factors are likely to be involved in this association.

The association between ovarian cancer and endometriosis has also been confirmed by case-control studies. Ness et al. produced findings in line with Brinton et al., reporting an OR of 1.73 (95\% CI 1.1-2.7) in women with endometriosis compared to controls. The underlining mechanisms seem to be attributable to immunological and hormonal factors. In particular, studies of endometriosis in patients with ovarian cancer reported a clear specific association to malignant histotype, endometrioid and clear cell carcinomas [1820].

Although the current findings are strong enough to support a link between endometriosis and ovarian cancer their association cannot be used to infer causality. They may indeed represent two distinct or two consequent entities as shown in Fig. 1.

\section{Breast cancer and infertility}

Several cohort studies of infertile women have reported an incidence of breast cancer similar to that of the general population, even without adjustment for parity, with SIR ranging from 0.9 to 1.4 $[1-3,6,12]$. The slightly higher incidence in infertile women may be related to the expected nulliparity and to late age at first birth. However, recently Jensen et al. reported a significantly increased SIR for breast cancer (SIR 1.08 95\% CI: 1.01-1.16) in a large Danish cohort, after adjustment for parity status [9].

Among the different causes of infertility, ovulation disorders, characterized by an unbalanced estrogen environment, would have expected to have a clear agreement vs higher risk, but the data are not really conclusive $[1-3,6]$.

Garland in a large prospective cohort of US women reported that a multivariate RR value of 0.41 associated with a self-reported history of ovulatory infertility. A history of infertility, excluding ovulatory infertility, also showed no increase in breast cancer risk with $\mathrm{RR}=1.06(95 \% \mathrm{CI} 0.76-1.48)$ [21]. These data are in line with another prospective study that reported similar risk of breast cancer ( $\mathrm{RR}=1.2 ; 95 \% \mathrm{CI}=0.7-2.0)$ in women affected by polycystic ovary syndrome and in controls [22]. Moreover, the recent prospective study by Terry et al. of infertility due to ovulatory disorders and incidence of breast cancer found a significantly lower breast cancer risk in women reporting infertility than in women who did not report infertility [23]. Case-control studies reported similar data with no increase in breast cancer risk in all infertility types as well as in ovulatory disorders infertility [24]. Data about association of breast cancer and endometriosis are instead inconclusive. The initial report of an increased risk in the Swedish cohort by Brinton et al. [16] was not confirmed by further studies [2]. The same is true for casecontrol studies $[25,26]$, reporting a significantly increased OR in premenopausal women, as if the two pathologies could share a common inflammatory pathway [27].

\section{Endometrial cancer and infertility}

Cancer of endometrium has been associated with infertility by a number of studies $[2,4,9,28-35]$. Comparing to the general population, two large cohort studies reported an increased risk of endometrial cancer in infertile patients. Venn found an SIR of 2.47 for untreated IVF clinic patients, and Modan of 4.8 in patients treated for infertility $[2,4]$. The recent large Danish cohort study found a $29 \%$ borderline-significant crude increased risk, but the risk was not significantly increased when results were adjusted for parity [9].

Among ovulatory disorders, the association between polycystic ovarian syndrome (PCOS) and endometrial cancer has been 
Endometriosis

Endometriotic cells

(ectopic endometrium)

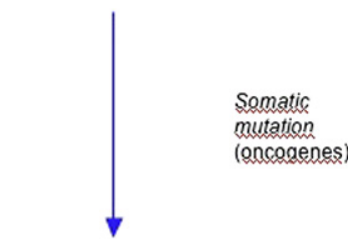

Epithelial ovarian cancer:

\section{Endometrioid}

Clear cell hustotyoe

\section{Common risk factors: \\ Genetic predisposition \\ Immunological dvsregulation \\ Environmental factors (inflammation) \\ Nulliparity \\ Early age at menarche \\ Regular menstrual cycle}

reported by several studies. PCOS is the most common ovulatory disorder and it is diagnosed in the presence of two or more of chronic anovulation, clinical or biochemical signs of hyperandrogenism, and polycystic ovaries (2003) Rotterdam criteria [28].

In PCOS, the prolonged anovulation and consequent release of estrogen unopposed by progesterone may enhance the development and growth of endometrial cancer, particularly in young women [29]. Hypersecretion of luteinizing hormone (LH), chronic hyperinsulinemia and increased serum insulin-like growth factor 1 (IGF-1) levels may represent additional risk factors for endometrial cancer [29], as shown in Fig. 2. An association between PCOS and endometrial cancer was suggested since 1949 by Speert [30] and in 1957 by Jackson and Docherty [31]. However, there is only little evidence to support a real association between endometrial cancer

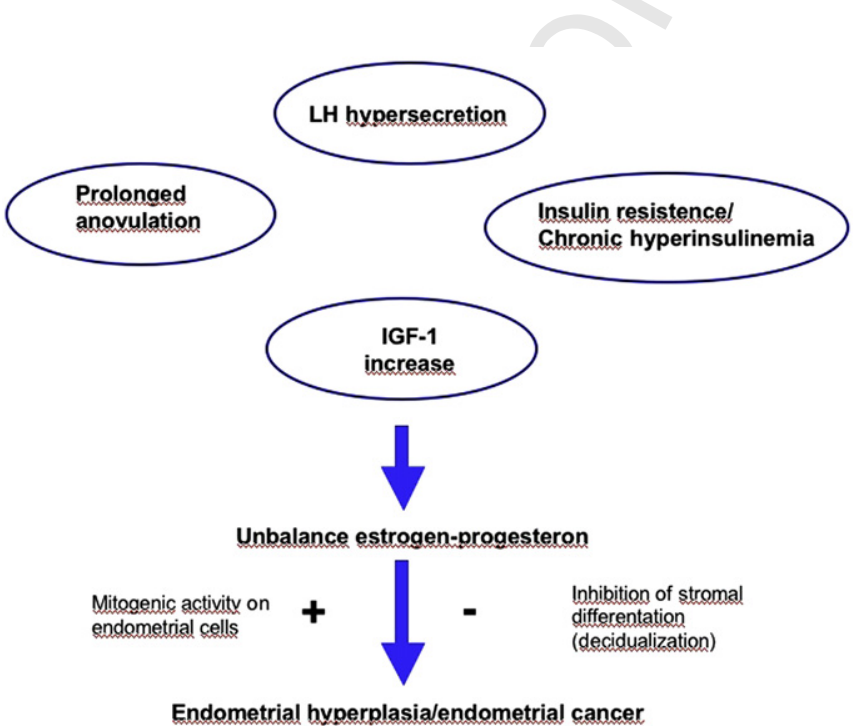

Fig. 2. Possible mechanisms implicated in the predisposition of polycystic ovarian syndrome and predisposition to endometrial cancer. and PCOS, and this evidence seems to be inconclusive [32]. Moreover, in a longitudinal study of 750 women with PCOS, mortality from EC was not increased [33] and the prognosis for endometrial cancer seemed better, since these cancers showed higher degrees of differentiation [34]. These data are not in agreement with Pillay's report, where the immunohistological evidence of the protooncogene Cyclin D1 seems to be more prevalent in endometrial cancer of women with PCOS [35].

At present, it is not possible to reach a definitive conclusion, since most studies have investigated the association between endometrial cancer and anovulatory infertility, rather than PCOS, while others have shown an association between PCOS and endometrial hyperplasia, assuming the data could be extended to endometrial cancer. Lastly, different definitions have been utilized for PCO/PCO Syndrome. A recent study by Pillay et al. [35] investigated the prevalence of polycystic ovary (PCO), as a marker of PCOS, in ovarian sections of women with endometrial cancer compared to patients with benign conditions. The authors concluded that PCO was similarly prevalent in the two groups; however, in women aged $<50$ years, PCO was more prevalent in women with endometrial cancer ( $62 \%$ vs $27 \%$ ). More than 50 years after an association between PCOS and endometrial cancer was first suggested, the nature of this association remains unclear [30,31].

\section{Other cancers}

The incidence of other extra-gynecological cancers has been investigated by several studies in women affected by infertility, and in particular by endometriosis. The incidence of invasive and in situ cervical cancers in infertile women has been found to be significantly lower than expected from general population incidence rates in two cohort studies [36,37]. These data may be explained by the increased number of referrals to gynecologist from infertile women, and the consequent more frequent cervical screening tests which allow greater detection and treatment of low-grade lesions before their progression to invasive cancer [16,36,37].

The incidence of melanoma has also been associated with infertility. Rossing et al. reported melanoma to be significantly 
increased in infertile women [38] but this association was not confirmed by other studies [37]. The most recent study by Brinton et al. [39] documented an increased twofold risk of melanoma in infertile women with endometriosis compared to patients with other causes of infertility.

An interesting recent finding, which needs further evidence to be confirmed, is also the possible association between endometriosis and Non-Hodgkin lymphoma reported by some studies $[16,17,39]$.

\section{Ovulation induction and cancer risk}

In the past years, much attention has been focused on the possible association between the use of fertility medications (FMs) and the development of malignancies of the ovary, breast, endometrium, and thyroid gland as well as of melanoma. A number of investigations have attempted to address the long-term effects of ovulation-inducing medications on cancer risk, but most have had shortcomings. These include small number of study subjects, short follow-up, imprecise information on drug exposures and indications for usage, and absence of information on other correlates of drug exposure that could influence cancer risk. Since hormonal and reproductive factors are known to be involved in the etiology of cancers of the female reproductive system, a stimulating effect of fertility medications on the risk of these cancers is theoretically possible. The precise mechanisms involved in the pathogenesis of hormone-related cancers remain unclear, and thus, it is difficult to predict how and to which extent FM may affect the risk of various cancers.

\subsection{Ovarian cancer and ovulation induction}

Ovarian cancer is the fifth most common malignancy in women in developed countries and accounts for approximately $4 \%$ of all malignancies in females. In general, the highest incidence rates of ovarian cancer are seen in North America and Scandinavia whereas the lowest rates are seen in Asia. The large majority of ovarian malignancies originates from the ovarian epithelium (80-90\%). Non-epithelial tumors of the ovary, such as germ cell tumors and sex-cord tumors, originate from the ovarian stroma and account for only $4-6 \%$ of all ovarian neoplasms.

To explain the epidemiology of epithelial ovarian cancer, three main hypotheses have been developed. First, ovarian cancer might be caused by repeated ovulations disrupting the ovarian epithelium and leading to malignant transformation of the epithelial cells $[40,41]$.

The second hypothesis proposes a model in which persistent stimulation of the ovary by gonadotropins increases the risk of malignant changes [42]. The third hypothesis proposes a carcinogenic role for exposure of the ovarian epithelium to environmental agents, such as talcum powder, that may enter the pelvic cavity through the vaginal canal. Talcum powder can be contaminated with asbestos minerals known to be associated with excess mortality from various cancers [43]. Recently, two new hypotheses have been postulated. One is the "endometriosis hypothesis" where endometriosis may act to promote the development of ovarian cancer if endometriotic implants cause irritation and subsequent inflammatory reactions [44]. The other hypothesis is that ovarian cancer may be increased by factors associated with excess androgenic stimulation of ovarian epithelial cells and may be decreased by factors related to greater progesterone stimulation.

Three lines of evidence raise concern regarding potential effects of ovulation-inducing medications on cancer risk. First the most commonly used medications, clomiphene citrate and gonadotropins, are effective for stimulating ovulation, a factor implicated in the etiology of both breast and ovarian cancers (Fig. 3). Second,

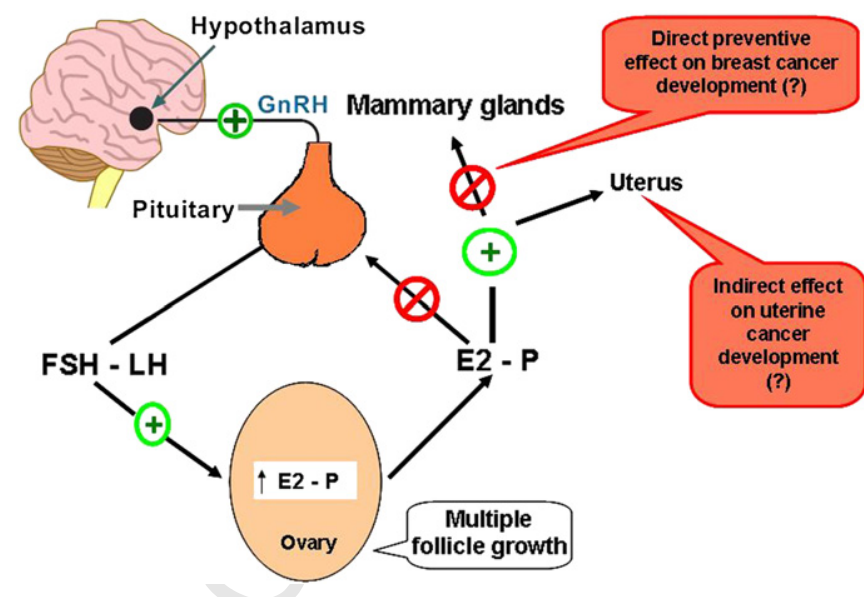

Fig. 3. Action of clomiphene and possible action on breast and endometrial cancers.

these medications raise both E2 and P levels, hormones that are recognized as affecting the development and growth of breast and gynecology cancers as well as some other cancers. Several clinical reports have suggested a potential relationship between the use of ovulation-inducing medications and ovarian cancer risk.

Rossing et al. [6] examined the risk of ovarian tumors in a cohort of 3837 women evaluated for infertility between 1974 and 1985 in Seattle. The authors found 11 invasive or borderline malignant ovarian tumors, with a relative risk of $2.5(95 \% \mathrm{CI}, 1.3-4.5)$. Although the risk of an invasive epithelial ovarian tumor was somewhat increased (age-standardized incidence ratio, 1.5; 95\% CI, 0.4-3.7), the risk of a borderline tumor was substantially higher than that expected on the basis of rates in the general population of women (age-standardized incidence ratio, 3.3; 95\% CI, 1.1-7.8). In particular, Rossing et al. [6] found that, although the risk was increased independently from the cause of infertility, it was roughly twice as likely to develop in women with ovulatory abnormalities than in the infertile women with other types of abnormalities. Moreover, the authors described that ovarian tumors developed in nine of the women who had used clomiphene, resulting in a higher risk than that among infertile women who had never used the drug, and that the women who used the drug for 12 or more cycles were at considerably increased risk (adjusted relative risk, 11.1; 95\% CI, 1.582.3). These data suggested that prolonged use of clomiphene may increase the risk of a borderline or invasive ovarian tumor.

However, the same authors [12] did not found an increased risk in a population-based, case-control study among women aged 3554 treated with ovulation-inducing medications, including 378 cases and 1637 controls. Both among parous and nulliparous women, the authors observed no association of cancer risk with a history of infertility, medical evaluation for infertility, specific types of infertility, or use of ovulation-inducing medications.

These contradictory data were reported also in a retrospective study performed by Brinton et al. [45] to assess the long-term effects of ovulation-stimulating medications on the risk of ovarian cancer. Studying 12,193 patients, Brinton et al. evidenced that infertile subjects had a significantly higher risk of developing ovarian cancer than the general population (standardized incidence ratio $1.98 ; 95 \% \mathrm{CI} 1.4,2.6$ ) and that the ovarian cancer risks were similar in patients unexposed and exposed to clomiphene or gonadotropins. To assess drug usage effects after accounting for other factors that might influence ovarian cancer risk, the same authors performed subsequent analyses on internal comparisons to derive adjusted rate ratios and found that dosage and number of cycles of FM were not associated with increased risk. Moreover, there were higher, although non-significant, risks with follow-up 
time, with rate ratios after 15 or more years of 1.48 (95\% CI 0.7, 3.2) for exposure to clomiphene (five exposed cancer patients) and 2.46 (95\% CI 0.7, 8.3) for gonadotropins (three exposed cancer patients).

Tables 1 and 2 present the principal studies reporting the relationship between the use of FM and ovarian cancer risk. Among these studies, the results of Rossing did depend on population data, whereas the studies of Brinton as well as Klip had internal comparison groups $[6,45,46]$.

Besides the attention pointed to the relationship between FM and ovarian cancer risk, other studies have concentrated on exposure during IVF programs. Venn et al. [2] studied the incidence of cancer in 29,700 women who had undergone IVF treatment and divided them into 20,656 patients exposed to fertility medications and 9044 not exposed. The authors reported that the ovarian cancer incidence was not greater than expected in the FM group (SIR 0.91; 95\% CI 0.74-1.13).

Similar results suggesting a low/no association between FM and ovarian cancers were also reported in 25,152 women undergoing IVF programs in the Netherlands [46] and in several studies characterized by small and selected populations [10,47-49].

In conclusion, although the uninterrupted ovulation induced by medications used for superovulation justifies the potential link between the use of fertility medications and ovarian cancer risk, the studies published reported reassuring results. In addition, the possible influence of dietary and genetic factors, hormonal status, parity and other risk factors, indicates the need for new studies characterized by a long follow-up and considering all confounding factors.

\subsection{Endometrial cancer and ovulation induction}

Endometrial cancer ranks fourth among diagnosed cancers, behind breast, lung, and bronchus and colon and rectum cancers. In most industrialized countries, cancer of the corpus uteri is about as frequent as ovarian cancer accounting for $6 \%$ of all new cancers. Throughout her lifetime, a woman has a 1 in 37 chance of developing endometrial cancer. Recognized risk factors for endometrial cancer are nulliparity, late age at menopause, obesity, PCOS, and presence of estrogen-secreting malignancies. Anovulation, infrequent ovulations, and various progesterone deficiencies mostly characterize hormonal subfertility. Irregular menstrual cycles are often anovulatory or have a prolonged follicular phase. Both features result in prolonged exposure to estrogen or progesterone and this might raise the risk of endometrial cancer.

Two types of endometrial cancer have been described. Type I is associated with hyperestrogenic states and expresses estrogen and progesterone receptors. Type II is not associated with hyperestrogenism and functional receptors are rarely expressed. Much like hormone-dependent breast cancer, the theory behind hormone-dependent endometrial cancer is that estrogen stimulates the mitotic activity of the endometrium, whereas it induces differentiation. Increased cell division increases the probability of random mutations, leading to cancer.

In 1994, Miannay et al. [50] described three cases of adenomatous hyperplasia of the endometrium, occurring among women treated with FM, but acknowledged the difficulty of establishing an association between these hormonal therapies and adenocarcinoma or its antecedents signs. In the same line of thought, most studies have not observed an association between fertility medications and endometrial cancer $[2,4,51]$ but most of these studies show the limit of a follow-up time of less than 10 years.

Venn et al. [2] reported the findings of a follow-up study of cancer incidence in 29,700 women who had referred for in vitro fertilization (IVF) treatment. Interestingly, in these studies the authors enrolled 20,656 women undergoing IVF treatment with ovarian stimulation and 9044 women undergoing IVF but without treatment cycles. Cancers of the uterus were no more common than expected in IVF treated women (five observed, 4.6 expected, SIR $1.09,95 \%$ CI $0.45-2.61$ ) but they were significantly more common in patients referred for IVF but not treated (seven observed, 2.8 expected: SIR 2.47, 95\% CI 1.18-5.18). In these studies cancers of the uterus were diagnosed in women aged 35-48 and included eight endometrial adenocarcinomas, two stromal sarcomas and three leiomyosarcomas. Interestingly, all sarcomas were found in untreated IVF patients.

Recently, two large studies have been performed studying the effects of fertility medications on endometrium. Among 2496 infertile Israeli women treated with clomiphene or clomiphene and human menopausal gonadotropins (hMG) between 1964 and 1974, Modan et al. [4] described 21 endometrial cancers vs 4.3 expected ( $\mathrm{SIR}=4.85,95 \% \mathrm{CI} 3.0-7.4)$, and evidenced that confounding by nulliparity, obesity, and contraceptive use or hysterectomy could not fully explain the increased risk ratio for endometrial cancer. The authors also reported that endometrial cancer was prominent among patients with normal estrogen production but progesterone deficiency (SIR $=9.4,95 \%$ CI 5.0-16.0).

Similarly, Althuis et al. [51] performed a retrospective cohort study of 8431 US women (145.876 woman-years) evaluated for infertility during 1965-1988 and described 39 uterine cancers. Analysis of patients by questionnaire or by cancer and death registries suggested that clomiphene may increase uterine cancer risk (rate ratio (RR): 1.79; 95\% confidence interval (CI): 0.9-3.4). In particular, women with an anovulatory disorder also were at elevated risk of uterine cancer, whereas other causes of infertility such as endometriosis, tubal disease, and male factor, uterine, or cervical disorders were not related to uterine cancer risk. Moreover, uterine cancer risk increased with clomiphene dose (RR: 1.93; 95\% CI: $0.9-4.0$ for $>900 \mathrm{mg}$ ), menstrual cycles of use (RR: 2.16 ; $95 \% \mathrm{CI}$ : 0.9-5.2 for $\geq 6$ cycles), and time elapsed since initial use (RR: 2.50 ; 95\% CI: 0.9-7.2 for women followed for $\geq 20$ years). Interestingly, the risk was more strongly associated with clomiphene among nulligravid (RR: 3.49; 95\% CI: 1.3-9.3) and obese (RR: 6.02 ; 95\% CI: 1.2-30.0) women.

In conclusion, the studies published as of today suggest an increased risk of endometrial cancer in infertile patients with hormonal defects where FM could accelerate cancer development. However, the short follow-up and the lack of information on important confounders, such as the cause of infertility or parity represent a great limitation of some of the studies analyzed.

\subsection{Breast cancer and ovulation induction}

Breast cancer is the most common malignancy in women in developed countries and accounts for 30-35\% of all malignancies in females. A one in eight women has the probability of developing breast cancer during her lifetime. Breast cancers can be divided into two groups: those whose growth is hormone dependent and those that are not responsive to hormones. In general, the hormoneresponsive tumors are estrogen receptor positive and these ERpositive tumors represent $60-75 \%$ of all breast cancer incidents. These data suggest that breast cancer is associated to a hormonal etiology and consequently ovulation induction medications could contribute to cancer development.

In the last years several cohort and case-control studies were performed to test this hypothesis. All these studies have not evidenced a greater risk in relation to fertility medications $[2,4,14,37,52-56]$, but they are limited by the small number of cancers evaluated.

Among these studies, Burkman et al. [57] performed a casecontrol study enrolling 4575 patients with histologically confirmed primary invasive breast cancer and 4682 control subjects without breast cancer identified in the same geographic locations. The 
authors suggested that a history of infertility drug use was not associated with the risk of developing breast cancer but evidenced that women using human menopausal gonadotropin (hMG) for $\geq 6$ months or for at least 6 cycles had a relative risk of breast cancer ranging between 2.7 and 3.8 .

Contradictory results were also reported in two large epidemiological studies performed in Australia [2] and Holland [46]. Both studies found no association between FM and cancer risk. However, Venn et al. [2] described a twofold increased risk of breast cancer within 1 year of last treatment, suggesting a possible role of fertility medications to promote, but not to induce, a preexisting cancer. Moreover, Gauthier et al. [58] reported that infertility treatment was associated with an increased risk, of borderline significance, of breast cancer among women with a family history of breast cancer.

Finally, some studies suggested a preventive effect of clomiphene on breast cancer development. Clomiphene is a selective estrogen receptor modulator (SERM) with similar properties to another SERM such as tamoxifen. Two studies reported a reduced risk in patients undergoing fertility medication with clomiphene $[59,60]$ and evidenced that the risk decreased significantly with duration of therapy. Contrary, Lerner-Geva et al. [61] observed 131 breast cancers in 5788 women attending five infertility centers in Israel between 1964 and 1984 and reported that the risk for breast cancer was significantly higher for women treated with clomiphene citrate $(\mathrm{SIR}=1.4 ; 95 \% \mathrm{CI} 1.0-1.8)$. These results reached statistical significance even when well-known risk factors for breast cancer (such as family history and cycle index) were controlled for, suggesting that clomiphene may have a direct antiestrogenic effects on the breast and that this effect may be overridden by the elevated estradiol levels induced by clomiphene in women of reproductive age.

Finally, Jensen et al. [9] analyzed a cohort of 54,362 women with infertility referred to all Danish fertility clinics between 1963 and 1998 and found 331 invasive breast cancers. Analyses within cohorts showed no overall increased breast cancer risk after use of gonadotropins, clomiphene, human chorionic gonadotropin, or gonadotropin-releasing hormone, whereas use of progesterone increased breast cancer risk (RR, 3.36; 95\% CI, 1.3-8.6). For all groups of fertility drugs, no relationships with number of cycles of use or years since first use of fertility drug were found. However, gonadotropins seemed to have a stronger effect on breast cancer risk among nulliparous women (RR, 1.69; 95\% CI, 1.03-2.77).

In conclusion, given that breast cancer is widely recognized as having a hormonal etiology, further assessment on the effects of fertility medications should be undertaken.

\subsection{Other cancers}

Melanoma is a cutaneous cancer and steroid hormones could be involved in its pathogenesis. The changes in the hormonal levels induced by fertility medications raise the question of possible effects of FM in melanoma development.

In the largest IVF follow-up study evaluating risk of melanoma in patients treated with fertility medications Klip et al. [46] reported 34 cases of melanoma and risk was not found to be increased in treated women. Other studies characterized by a small number of melanomas observed (Rossing et al. [38]: 12 cases; Venn et al. [2]: 12 cases; Young et al. [62]: 14 cases) reported similar results.

The greater incidence of thyroid cancer in women than men may imply that female hormones are involved in the etiology of thyroid cancer. Consequently some studies were performed to assess fertility medications' influence in thyroid cancer. In a retrospective cohort of 8422 women, Althius et al. [63] reported that clomiphene or gonadotropins' use did not significantly increase risk of thyroid cancer. Similar results were reported also by Kolonel et al. [64] and La Vecchia et al. [65]. Recently, Hannibal et al. [66] performed a follow-up study in 54,362 women with infertility and identified 29 thyroid cancers. The authors evidenced that use of clomiphene was associated with an increased risk of thyroid cancer $(\mathrm{RR}=2.28$; 95\% $\mathrm{CI}: 1.08-4.82)$, suggesting that longer follow-up studies are needed to obtain conclusive results.

\section{Limitations of studies assessing infertility and cancer risk}

Whether infertile women are at increased risk of cancer due to their infertility or factors such as ovulation medication induction, has been the subject of several studies. Studies examining whether infertility itself or ovulation induction are associated with an increased risk have met considerable challenges. It is important to consider these limitations in interpreting different studies reports: differences of some studies may reflect the possible variability in population selection. Overall, the limitations of these findings include difficulties in achieving an adequate sample size, accurate diagnosis of infertility, or data about treatment with infertility medications with the respective dosages and duration exposure. The interpretation of findings have to consider potential confounding risk factors that need to be identified and controlled such as age at menarche, history of contraceptive use or hormone replacement therapy, parity, age at first birth, family history of breast and ovarian cancer. Moreover, there is no study to our knowledge, that has addressed potential differences between gravidity or parity as confounding factors for cancer development in infertile women.

Available studies can be generally subdivided in cohort or casecontrol design. They both present advantages and disadvantages to address this question.

Most cohort studies have selected their population from patients from infertility clinics and as such reflecting cancer risk in a specific population of women seeking medical treatment for infertility. Cohort studies present the advantage that accurate information about exposure to ovulation induction medications is available from clinical records. A possible bias which should be taken into account in interpreting these results is that the collected data are specifically referred to the medical stimulation in that clinic, ignoring possible treatments in other medical settings and, therefore, further data in terms of total number of cycle, type and duration of medical treatment could be omitted. Therefore, these studies cannot be generalized to all populations. Moreover, in some cohort studies data about other potential biases that could arise later, such as parity, contraceptive use, family history of breast or ovarian cancer, could be omitted. Parity is the most relevant confounder to adjust and stratify for, as the frequency of nulliparity is higher among infertile women than among fertile women. Otherwise, the cancer risk among infertile women will be overestimated as a consequence of the increased risk in nulliparous women. Furthermore, most cohort studies present the inevitable limitation of including a small number of cancer cases due to young age for cancer development with short periods of follow-up. Several cohort studies use the standardized incidence ratios (SIRs) to compare cancer risk in infertile women with that of the general population. This is a statistical parameter difficult to interpret because of the inability to control for other factors that might distinguish cancer risks for infertile women.

Case-control studies populations are selected from hospitals in some instances and from population-based registries in others. They are limited by their ability in defining and analyzing separately patients with different causes of infertility. Moreover, they rely on self-reported fertility drug use, which can be easily subject to error when recalled after many years. Some studies have determined the cause of infertility from medical records, but the accuracy of data may vary according to infertility investigations, clinical interpretation and definition of disease severity by the 
different centers. However, differently from cohort studies most case-control studies offer results corrected for parity.

In general, studies on cancer risk associated with infertility or medications' use are limited by low statistical power, in particular when proposing a sub-analysis of infertility or type and time of infertility treatment. Multicenter studies or pooled data by different reports usually use common definition of population characteristics and modalities of recording data and reach adequate sample size to give a precise estimation of the problem $[10,11]$. Meta-analysis do not offer a similar value, since they pool together findings of individual heterogeneous studies, risking to add too much bias in the final considerations.

\section{Conclusions}

Epidemiological studies have suggested a possible association between infertility, fertility drug use, and increased cancer risk. Although findings are generally reassuring, nowadays we have no firm answers in counseling infertile couples. Current evidence is mostly based on case-control and cohort studies, which offer an estimate of the problem. Larger population studies, better adjustment for confounding factors, such as parity, infertility, contraceptive use, early age at menarche, and late age at menopause, which coexist in infertile patients, and long-term follow-up may offer more precise data in the future. In particular, studies are needed to better understand the patho-physiological mechanisms underlying the apparent association between ovarian cancer and infertility, as well as the association with endometriosis.

\section{References}

[1] Brinton LA, Melton III LJ, Malkasian Jr GD, Bond A, Hoover R. Cancer risk after evaluation for infertility. Am J Epidemiol 1989;129:712-22.

[2] Venn A, Watson L, Bruinsma F, Giles G, Healy D. Risk of cancer after use of infertility drugs with in-vitro fertilization. Lancet 1999;354:1586-90.

[3] Potashnik G, lerner-geva L, Genkin L, Chetrit A, Lunenfeld E, Porath A. Fertility drugs and the risk of breast and ovarian cancers: results of a long-term followup study. Fertil Steril 1999;71:853-9.

[4] Modan B, Ron E, Lerner-Geva L, Blumstein T, Menczer J, Rabinovivi J, et al. Cancer incidence in a color of infertile women. Am J Epidemiol 1998;147:1038-42.

[5] Brinton LA, Lamb EJ, Moghissi KS, Scoccia B, Althuis MD, Mabie J, et al. Ovarian cancer risk associated with varying causes of infertility. Fertil Steril 2004;82:405-14.

[6] Rossing MA, Daling JR, Weiss NS, Moore DE, Self SG. Ovarian tumors in a cohort of infertile women. N Engl J Med 1994;331:771-6.

[7] Harlow BL, Weiss NS, Roth GS, Chu J, Daling JR. Case-control study of borderline ovarian tumors: reproductive history and exposure to exogenous female hormones. Cancer Res 1988;48:5849-52.

[8] Tworoger SS, Fairfield KM, Colditz GA, et al. Association of oral contraceptive use, other contraceptive methods, and infertility with ovarian cancer risk. Am J Epidemiol 2007;166:894-901.

[9] Jensen A, Sharif H, Jorgen HO, Kruger Kjaer S. Risk of breast cancer and gynecologic cancers in a large population of nearly 50,000 infertile Danish women. Am J Epidemiol 2007;16(7):1400-7.

[10] Whittemore A, Harris R, Itnyre J. The collaborative Ovarian Cancer Group. Characteristics relating to ovarian cancer risk: collaborative analysis of 12 US case-control studies. II. Invasive epithelial ovarian cancers in white women. Am J Epidemiol 1992;135:1184-203.

[11] Ness RB, Cramer DW, Goodman MT, Kjaer SK, Mallin N, Mosgaard BJ, et al. Infertility, fertility drugs, and ovarian cancer: a pooled analysis of case-control studies. Am J Epidemiol 2002;155:217-24.

[12] Rossing MA, Tang MT, Flagg EW, Weiss LK, Wicklund KG. A case-control study of ovarian cancer in relation to infertility and the use of ovulation induction drugs. Am J Epidemiol 2004;160:1070-8.

[13] Schildkraut JM, Schwingl PJ, Bastos E, Evanoff A, Hughes C. Epithelial ovarian cancer risk among women with polycystic ovary syndrome. Obstet Gynecol 1996;88:554-9.

[14] Ron E, Lunenfeld B, Menczer J, Blumemstein T, katz L, Oelsner G, et al. Cancer incidence in a cohort of infertile women. Am J Epidemiol 1987;125:780-90.

[15] Melin A, Sparen P, Bergquist A. The risk of cancer and the role of parity among women with endometriosis. Hum Reprod 2007;22:3021-6.

[16] Brinton LA, Gridley G, Person I, Baron J, Bergqvist A. Cancer risk after a hospital discharge diagnosis of endometriosis. Am J Obstet Gynecol 1997;176:572-9.

[17] Berglund AS, Sparen O, Bergqvist A. Endometriosis and the risk of ovarian cancer. Abstract of the 19th Annual Meeting of ESHRE, Madrid, Hum Reprod, Abstract Book; 2003.
[18] Vercellini P, Parazzini F, Bolis G, Carinelli S, Dindelli M, Vendola N, et al. Endometriosis and ovarian cancer. Am J Obstet Gynecol 1993;169:181-2.

[19] Ogawa S, Kuku T, Amada S, Kobayashi H, Hirakawa T, Ariyoshi K, et al. Ovarian endometriosis associated with ovarian carcinoma: a clinic-pathological and immunohistochemical study. Gynecol Oncol 2000;77:298-304.

[20] Oral E, Ilvan S, Tustas E, Korbeyli B, Bese T, Demirkiran F, et al. Prevalence of endometriosis in epithelial ovary tumors. Eur J Obstet Gynecol Reprod Biol 2003;109:97-101.

[21] Garland M, hunter DJ, Coldhits GA, Manson JE, Stampfer MJ, Spiegelman D, et al. Menstrual cycle characteristics and history of ovulation infertility in relation to breast cancer risk in a large cohort of US women. Am J Epidemiol 1998; 147:636-42.

[22] Anderson KE, Sellers TA, Chen P-L, Rich SS, Hong CP, Folsom AR. Association of Stein-Leventhal syndrome with the incidence of postmenopausal breast carcinoma in large prospective study of women in IOWA. Cancer 1997;79:494-9.

[23] Terry KL, Willet WC, Rich-Edwards JW, et al. A prospective study of infertility due to ovulatory disorders, ovulation induction, and incidence of breast cancer. Arch Intern Med 2006;166:2484-9.

[24] Gammon MD, Thompson WD. Polycystic ovaries and the risk of breast cancer Am J Epidemiol 1991;134:818-24.

[25] Moseson M, Koenig KL, Shore RE, Pasternack BS. The influence of medical conditions associated with hormones on the risk of breast cancer. Int J Epidemiol 1993;22:1000-9.

[26] Weiss HA, Brinton LA, Potishman NA, Brogan D, Coates RJ, Gammon MD, et al Breast cancer risk in young women and history of selected medical conditions. Int J Epidemiol 1999;28:816-23.

[27] Ness RB, Modugno F. Endometriosis as a model for inflammation-hormone interactions in ovarian and breast cancer. Eur J Cancer 2006;42:691-703.

[28] Rotterdam ESHRE/ASRM-Sponsored PCOS Consensus Workshop Group. Revised 2003 consensus on diagnostic criteria and long-term health risks related to polycystic ovary syndrome (PCOS). Hum Reprod 2004; $19: 41-7$

[29] Giudice LC. Endometrium in PCOS: implantation and predisposition to endocrine CA. Best Pract Res Clin Endocrinol Metab 2006;20:235-44.

[30] Speert H. Carcinoma of the endometrium in young women. Surg Gynecol Obstet 1949;88:332-6.

[31] Jackson RL, Docherty MB. The Stein-Leventhal syndrome: analysis of 43 cases with special references to association with endometrial carcinoma. Am J Obstet Gynecol 1957;73:161-73.

[32] Hardiman P, Pillary OC, Atiomo W. Polycystic ovary syndrome and endometrial carcinoma. Lancet 2003;361:1810-2.

[33] Peirpoint T, McKeigue PM, Isaacs AJ, Wild SH, Jacobs HS. Mortality of women with polycystic ovary syndrome at long-term follow-up. J Clin Epidemiol 1998;51:581-6.

[34] Jafari K, Javaheri G, Ruiz G. Endometrial adenocarcinoma and the SteinLeventhal Syndrome. Obstet Gynecol 1978:51:97-100.

[35] Pillay OC, Wong Te Fong LF, Crow JC, Benjiamin E, Mould T, Atiomo W, et al. The association between polycystic ovaries and endometrial cancer. Human Reprod 2006;21:924-9.

[36] Rossing M, Daling J, Weiss N, Moore D, Self S. In situ and invasive cervical carcinoma in a cohort of infertile women. Fertil Steril 1996:65:19-22.

[37] Venn A, Watson L, Lumley J, Giles G, King C, Healy D. Breast and ovarian cancer incidence after infertility and in vitro fertilization. Lancet 1995;346:995-1000

[38] Rossing M, Daling J, Weiss N, Moore D, Self S. Risk of cutaneous melanoma in a cohort of infertile women. Melanoma Res 1995;5:123-7.

[39] Brinton LA, Westhoff CL, Scoccia B, Lamb EJ, Althiuus MD, Mabie JE, et al Causes of infertility as predictors of subsequent cancer risk. Epidemiology 2005;11:111-7.

[40] Booth M, Beral V, Smith P. Risk factors for ovarian cancer: a case-control study Br J Cancer 1989;60:592-8.

[41] Fathalla MF. Incessant ovulation - a factor in ovarian neoplasia? Lancet 1971;2:163.

[42] Risch HA. Hormonal etiology of epithelial ovarian cancer, with a hypothesis concerning the role of androgens and progesterone. J Natl Cancer Inst 1998:90:1774-86

[43] Cook LS, Kamb ML, Weiss NS. Perineal powder exposure and the risk of ovarian cancer. Am J Epidemiol 1997;145:459-65.

[44] Paulson RJ. Fertility drugs and ovarian epithelial cancer: the endometriosis hypothesis. J Assist Reprod Genet 1997;14:228-30.

[45] Brinton LA, Lamb EJ, Moghissi KS, Scoccia B, Althuis MD, Mabie JE, et al. Ovarian cancer risk after the use of ovulation-stimulating drugs. Obstet Gynecol 2004 Jun;103(6):1194-203.

[46] Klip H, Burger CW, Leeuwen Van, the Omega Project Group. Risk of hormone-related cancers after ovarian stimulation for in-vitro fertilization in a cohort of 25,152 women. In: Klip H, editor. Long-term health effects of subfertility treatment. the Netherlands: PrintPartners Ipskamp B.V. Enschede; 2002. p. 55-82.

[47] Shu XO, Brinton LA, Gao YT, Yuan JM. Population-based case-control study of ovarian cancer in Shanghai. Cancer Res 1989 July 1;49(13):3670-4.

[48] Franceschi S, La Vecchia C, Negri E, Guarneri S, Montella M, Conti E, et al. Fertility drugs and risk of epithelial ovarian cancer in Italy. Hum Reprod 1994 Sep;9(9):1673-5.

[49] Parazzini F, Negri E, La Vecchia C, Moroni S, Franceschi S, Crosignani PG. Treatment for infertility and risk of invasive epithelial ovarian cancer. Hum Reprod 1997 Oct;12(10):2159-61. 
[50] Miannay E, Boutemy JJ, Leroy-Billiard M, Gasnault JP, Leroy JL. The possible endometrial risk of ovarian stimulation. Apropos of 3 cases. J Gynecol Obstet Biol Reprod (Paris) 1994;23(1):35-8.

[51] Althuis MD, Moghissi KS, Westhoff CL, Scoccia B, Lamb EJ, Lubin JH, et al. Uterine cancer after use of clomiphene citrate to induce ovulation. Am J Epidemiol 2005 Apr 1;161(7):607-15.

[52] Doyle P, Maconochie N, Beral V, Swerdlow AJ, Tan SL. Cancer incidence following treatment for infertility at a clinic in the UK. Hum Reprod 2002;17:2209-13.

[53] Lerner-Geva L, Geva E, Lessing J, Chetrit A, Modan B, Amit A. The possible association between in vitro fertilization treatments and cancer development. Int J Gynecol Cancer 2003;13:23-7.

[54] Braga C, Negri E, La Vecchia C, Parazzini F, Dal Masso L, Franceschi S. Fertility treatment and risk of breast cancer. Hum Reprod 1996:11:300-3.

[55] Ricci E, Parazzini F, Negri E, Marsico S, La Vecchia C. Fertility drugs and the risk of breast cancer. Hum Reprod 1999;14:1653-5.

[56] Weiss HA, Troisi R, Rossing MA, Brogan D, Coates RJ, Gammon MD, et al. Fertility problems and breast cancer risk in young women: a case-control study in the United States. Cancer Causes Control 1998;9:331-9.

[57] Burkman RT, Tang MT, Malone KE, Marchbanks PA, McDonald JA, Folger SG et al. Infertility drugs and the risk of breast cancer: findings from the National Institute of Child Health and Human Development Women's Contraceptive and Reproductive Experiences Study. Fertil Steril 2003 Apr;79(4):844-51.

[58] Gauthier E, Paoletti X, Clavel-Chapelon F, E3N group. Breast cancer risk associated with being treated for infertility: results from the French E3N cohort study. Hum Reprod 2004 Oct;19(10):2216-21.
[59] Rossing MA, Daling JR, Weiss NS, Moore DE, Self SG. Risk of breast cancer in a cohort in infertile women. Gynecol Oncol 1996 Jan;60(1):3-7.

[60] Terry KL, Willett WC, Rich-Edwards JW, Michels KB. Clomiphene citrate use and reduced incidence of premenopausal breast cancer. Am J Epidemiol 2004;159(Suppl. 66):2615.

[61] Lerner-Geva L, Keinan-Boker L, Blumstein T, Boyko V, Olmar L, Mashiach S, et al. Infertility, ovulation induction treatments and the incidence of breast cancer - a historical prospective cohort of Israeli women. Breast Cancer Res Treat 2006 Nov;100(2):201-12 [Epub 2006 May 10].

[62] Young P, Purdie D, Jackman L, Molloy D, Green A. A study of infertility treatment and melanoma. Melanoma Res 2001;11:535-41.

[63] Althuis MD, Scoccia B, Lamb EJ, Moghissi KS, Westhoff CL, Mabie JE, et al. Melanoma, thyroid, cervical, and colon cancer risk after use of fertility drugs. Am J Obstet Gynecol 2005 Sep;193(3 Pt 1):668-74.

[64] Kolonel LN, Hankin JH, Wilkens LR, Fukunaga FH, Hinds MW. An epidemiologic study of thyroid cancer in Hawaii. Cancer Causes Control 1990;1:223-34.

[65] La Vecchia C, Ron E, Franceschi S, Dal Maso L, Mark SD, Chatenoud L, et al. A pooled analysis of case control studies of thyroid cancer III, oral contraceptives, menopausal replacement therapy and other female hormones. Cancer Causes Control 1999;10:157-66.

[66] Hannibal CG, Jensen A, Sharif H, Kjaer SK. Risk of thyroid cancer after exposure to fertility drugs: results from a large Danish cohort study. Hum Reprod 2008 Feb;23(2):451-6. 